\title{
Preventive effects of total saponins of Panax japonicus on fatty liver fibrosis in mice
}

\author{
Ding Yuan ${ }^{1,2}$, Tingting Xiang ${ }^{1}$, Yuanxiu Huo ${ }^{1}$, Chaoqi Liu' ${ }^{1}$, Ting Wang ${ }^{1}$, Zhiyong Zhou', \\ Yaoyan Dun ${ }^{1}$, Haixia Zhao ${ }^{1}$, Changcheng Zhang ${ }^{1}$
}

${ }^{1}$ Medical College of China Three Gorges University, Yichang, China
${ }^{2}$ Renhe Hospital of China Three Gorges University, Yichang, China

Submitted: 21 September 2015

Accepted: 14 December 2015

Arch Med Sci 2018; 14, 2: 396-406

DOI: $10.5114 /$ aoms.2016.63260

Copyright $\odot 2016$ Termedia \& Banach

\author{
Corresponding author: \\ Changcheng Zhang \\ Medical College \\ of China Three Gorges \\ University \\ Yichang, China \\ Phone/fax: \\ +8607176397366 \\ E-mail: greatwall@ctgu.edu.cn
}

\section{Abstract}

Introduction: Nonalcoholic fatty liver disease (NAFLD) is a condition in which excess fat accumulates in the liver of a patient without a history of alcohol abuse. Fatty liver fibrosis, a severe form of NAFLD, is a key step which can be reversed by effective medical intervention. This paper aims to describe the protective role and mechanisms of action of total saponins of Panax japonicus (SPJ) against fatty liver fibrosis in mice. In this study, fatty liver fibrosis was induced by a high-fat (HF) diet combined with intraperitoneal injection of porcine serum.

Material and methods: The fatty liver fibrosis model was induced by HF diet combined with intraperitoneal injection of porcine serum. The endoplasmic reticulum stress (ERS) response and C/EBP homologous protein (CHOP) and p-Jun $\mathrm{N}$-terminal kinase (JNK)-mediated apoptosis and inflammation were assessed by serum biochemistry, hematoxylin-eosin $(H+E)$, Masson and electronic microscopy staining, Hyp content detection, Western blotting and real time polymerase chain reaction (RT-PCR).

Results: Saponins of Panax japonicus could significantly improve liver function and decrease the lipid level in the serum. The liver steatosis, collagen fibers and inflammatory cell infiltration were significantly improved in the SPJ group according to microscope observation. The RT-PCR analysis revealed that the collagen I (Coll), $\alpha$ smooth muscle actin ( $\alpha$-SMA), tissue inhibitors of MMPs (TIMP), CHOP and GRP78 mRNA expression levels were distinctly weakened by SPJ treatment; and western blotting analysis indicated that the phosphorylated JNK ( $p-J N K)$, Coll and $78 \mathrm{kD}$ glucose-regulated protein (GRP78) protein expression levels were significantly alleviated, which might be associated with the inhibition of the ERS response and the CHOP and JNK-mediated apoptosis and inflammation pathway.

Conclusions: Based on this research, SPJ as a preventive medicine has great potential in prevention of liver fibrosis.

Key words: total saponins of Panax japonicus, fatty liver fibrosis, Coll, endoplasmic reticulum stress, JNK.

\section{Introduction}

As the lifestyle changes in the population worldwide, fatty liver diseases are gradually increasing in incidence [1] and have become the second most severe type of liver diseases after viral hepatitis [2]. Although fatty liver is considered to be a benign disease, without treatment it gradually develops into inflammatory cell infiltration and necrosis, which could 
result in liver fibrosis and cirrhosis and lead to a malignant disease, such as hepatocellular carcinoma [3]. It is of vital importance to find effective treatments to prevent and reverse the occurrence and development of fatty liver diseases.

Panax japonicus (PJ) belongs to the family Araliaceae in the plant kingdom. It is one of the very common traditional Chinese medicines, which grows wild throughout the southwest region of China and Japan. Saponin of Panax japonicus (SPJ) is the main ingredient, which can significantly inhibit inflammation and prevent liver injury in mice [4].

Here we aim to explore the preventive effects and the mechanism of action of total SPJ on fatty liver fibrosis in mice.

\section{Material and methods}

\section{Chemicals and reagents}

Authentic standards of Panax saponins Re, chikusetsusaponin V, chikusetsusaponin IV, chikusetsusaponin IVa, Pjs-2 and so forth were purchased from the National Institute of Control of Pharmaceuticals and Biological Products (Beijing, China). The hydroxyproline (Hyp) assay kit was purchased from Nanjing Jiancheng Bioengineering (Nanjing, China). The total protein extraction kit and BCA protein assay kit were purchased from Beijing Applygen Technologies Inc (Beijing, China). Rabbit polyclonal anti- $\beta$-actin antibody was bought from Wuhan Guge Biological Technology Co., Ltd. Anti-collagen I and GRP78 antibody were provided by Santa Cruz Biotechnology, Inc (Delaware, USA). Anti-phospho-SAPK/JNK and SPAK/JNK were purchased from Cell Signaling Technology (Danvers, USA). The total RNA extraction kit, retrovirus kit and PCR amplification kit were bought from TaKaRa Bioengineering Co., Ltd. (Dalian, China). Collagen I (Coll), a smooth muscle actin ( $\alpha$-SMA), tissue inhibitors of MMPs (TIMP), C/EBP homologous protein (CHOP), 78 kD glucose-regulated protein (GRP78), and glyceraldehyde-3-phosphate dehydrogenase (GAPDH) primers were purchased from Sangon Biological Engineering Co., Ltd. (Shanghai, China).

\section{Preparation of PJS and high-pressure liquid chromatography (HPLC) analysis}

The PJ was collected from Enshi Chunmuying Medicinal Material Planting Base, and authenticated by Dr Kun Zou, Hubei Key Laboratory of Natural Products Research and Development (China Three Gorges University). The SPJ were extracted according to the method of our research team [5]. Briefly, the root of dried Panax japonicus ( $1 \mathrm{~kg})$ was ground and then extracted three times with 10 l of $60 \%$ ethanol by recirculation for $2 \mathrm{~h}$. The extracted liquid was concentrated under reduced pressure to obtain a crude ethanol extract, which was suspended in $\mathrm{H}_{2} \mathrm{O}$, and then partitioned with $n$-butanol to obtain the $n$-butanol portion. The $n$-butanol extract was redissolved in water and poured into a chromatographic column containing macroporous adsorption resin D101 (Tianjin Pesticide Factory, China), rinsed with water until colorless and then eluted with different concentrations of ethanol in water. The $90 \%$ fraction was defined as the refined $n$-butanol extract, which was redissolved in water at a concentration of $10 \mathrm{mg} / \mathrm{ml}$ and then diluted with methanolwater to $1 \mathrm{mg} / \mathrm{ml}$. HPLC analysis was performed on a SEYMC-Pack ODS-AQ column (4.6 $\mathrm{mm} \times$ $250 \mathrm{~mm}, 5 \mu \mathrm{m}$ ) eluted with the mobile phases of acetonitrile (A) and $0.4 \%$ phosphate solution (B) at a flow rate of $1.0 \mathrm{ml} / \mathrm{min}$. The temperature of the column was $30^{\circ} \mathrm{C}$. The system was run with the following gradient program: $0-5 \mathrm{~min}$, 5\%A; 5-20 min, 5-30\%A; 20-30 min, 30\%A; 30$50 \mathrm{~min}, 30-85 \% \mathrm{~A} ; 50-60 \mathrm{~min}, 85 \% \mathrm{~A}$. The sample injection volume was $10 \mu \mathrm{l}$ and the detection was performed with a UV detector.

\section{Animal experiment}

The SPF level BABL/c mice (18-22 g, 4-5 weeks) with half males and half females were provided by Hubei Province Experimental Animal Center (number of animal license SCXK 2008-0005). Six mice were raised in a cage. All the animals were housed under standard conditions with a $12 \mathrm{~h}$ dark/light cycle at a temperature of $22 \pm 2^{\circ} \mathrm{C}$ and a humidity of $60 \pm 5 \%$. All of them were fed with standard rodent animal diet and water ad libitum. All procedures involving animals complied with the China National Institutes of Healthy Guidelines for the Care and Use of Laboratory Animals. All of this study's protocols were approved by the Animal Ethics Committee of the Three Gorges University.

A total of $48 \mathrm{BABL} / \mathrm{c}$ mice were randomly divided into four groups $(n=12)$ : the normal control group, the model group, the SPJ low dose group, and the SPJ high dose group. All of the mice were given high-fat (HF) forage except for the normal control group, each $10 \mathrm{~g}$ a day. At the same time, SPJ low and high dose groups were given a gavage of SPJ $100 \mathrm{mg} / \mathrm{kg}$ and $300 \mathrm{mg} / \mathrm{kg}$ respectively, once two days for 11 weeks. From the fifth week, the mice were injected intraperitoneally with pig serum, each $0.1 \mathrm{ml}$ a week four times, except for the control group, injected with PBS. The weight of the mice was weighed once a week at the same time. At the end of the $11^{\text {th }}$ week, all the mice stopped feeding for about $12 \mathrm{~h}$, and were anesthetized to collect the blood sample and then sacrificed to obtain the livers. The fresh liver was weighed to calculate the liver index (liver index $(\%)=$ liver weight/body weight $\times 100$ ). Then the 
liver was cut into slices and fixed for the histological and electron microscopic investigation. The remaining liver tissue was snap-frozen in liquid nitrogen and stored at $-80^{\circ} \mathrm{C}$.

\section{Serum biochemistry}

The serum activities of alanine aminotransferase (ALT), aspartate aminotransferase (AST), and the contents of triglyceride (TG), high-density lipoprotein $(\mathrm{HDL})$, low-density lipoprotein $(\mathrm{LDL})$, and total bile acid (TBA) were detected by professional testing kits according to corresponding product specifications.

\section{Histopathological examination}

The same parts of the liver tissue were chosen for hematoxylin-eosin $(H+E)$, Masson and electronic microscopy staining. Each staining method was performed according to the manufacturer's instructions. Briefly, tissue slices of $5 \mu \mathrm{m}$ thickness were made from the formalin-fixed specimens, and were stained with $\mathrm{H}+\mathrm{E}$ or Masson staining. For electronic microscopy staining, the liver tissues, about $1 \mathrm{~mm}^{3}$ in each mouse, were fixed with $2 \%$ glutaraldehyde overnight and washed three times with $0.2 \mathrm{M}$ phosphate buffer. Then the tissues were fixed again with $1 \%$ osmium tetroxide, washed with $0.2 \mathrm{M}$ phosphate buffer, and dehydrated with ethanol at different concentrations. The samples were immersed in Epon812 resin/ acetone $(1: 1)$ for $30 \mathrm{~min}$, and in fresh Epon812 resin for $30 \mathrm{~min}$, embedded for convergence overnight at $70^{\circ} \mathrm{C}$. The tissue was cut at about $50 \mathrm{~nm}$ thickness, stained with a negative-staining methodology with $2 \%$ uranyl acetate, and observed on a transmission electron microscope (TEM; H-7500, Hitachi, Tokyo, Japan).

\section{Determination of hydroxyproline content}

The hydroxyproline content was measured according to the instructions of the Hyp content detection kit.

\section{Detection of mRNA by real time-polymerase chain reaction}

The mRNA expression of the fibrogenesis related genes, $\alpha$-SMA and Coll, was determined by semi-quantitative RT-PCR. The housekeeping gene GAPDH was used as an internal control. Moreover, we also examined the gene expressions of TIMP, CHOP, and GRP78. Total RNA was extracted from snap-frozen liver tissue samples following the instructions provided with the total RNA extraction kit. The total RNA was reverse-transcribed with the reverse transcriptase kit according to the manufacturer's guidelines. The primers used for PCR are as in Table I. The expression levels of GAPDH were used as an internal control. Initial denaturation was performed at $94^{\circ} \mathrm{C}$ for $5 \mathrm{~min}$, followed by $25-30$ cycles of denaturing at $94^{\circ} \mathrm{C}$ for $30 \mathrm{~s}$, annealing at $55^{\circ} \mathrm{C}$ for $30 \mathrm{~s}$, and extending at $72^{\circ} \mathrm{C}$ for $30 \mathrm{~s}$.

\section{Western blotting analysis}

Total proteins were extracted using a total protein extraction kit according to the instructions. Protein concentrations were quantified using a BCA protein assay kit. Samples (50 $\mu$ g protein each) were separated by $10 \%$ SDS-polyacrylamide gel electrophoresis (PAGE) and transferred to polyvinylidene fluoride (PVDF) membranes by electrophoretic transfer. After being blocked in $5 \%$ non-fat milk in TBST for $1 \mathrm{~h}$ at room temperature, membranes were incubated with anti- $\beta$-actin antibodies (1:1000), anti-collagen I antibody (1 : 500), anti-GRP78 antibody (1 : 500), antip-JNK (1: 1000), and anti-JNK antibody (1:1000) at $4^{\circ} \mathrm{C}$ overnight. Immunoblots were incubated with the corresponding secondary antibody conjugated with horseradish peroxidase for several minutes. Membranes were developed using an electrochemiluminescence (ECL) kit. The density of the immunoreactive bands was analyzed using Image J 2x (National Institutes of Health, Bethesda, MD, USA).

Table I. Primer sequences used for real-time PCR assay

\begin{tabular}{|lcc|}
\hline Primer & Sequence $\left(5^{\prime} \rightarrow 3^{\prime}\right)$ \\
\cline { 2 - 3 } & Forward & Reverse \\
\hline$\alpha$-SMA & GGATTGACCCTAACCAAGGATGT & GGCTGCCACCATTGATAGTCTCT \\
\hline TIMP & TTGGGATGGAGTCAGCGG & TCCTGTCAGCAATGCCTGG \\
\hline CHOP & CTTCTTGGTTCCCTGGCGT & GCAAGTGACGGCTCTGGTAG \\
\hline GRP78 & TCACTGACTTGGGTTTGGGCTAT & TCAGACGGCTGTTCTCACTCCTA \\
\hline GAPDH & CGTGCGTGACATCAAAGAGAA & TGGCCACAGGATTCCAT \\
\hline
\end{tabular}




\section{Statistical analysis}

All data are expressed as means \pm standard deviation $(x \pm S)$. Statistical testing was performed with the SPSS 13.0 for Windows software. Data were analyzed by homogeneity of variances and one-way analysis of variance (ANOVA). P-values less than 0.01 were considered statistically significant.

\section{Results}

\section{Extraction and analysis of SPJ}

According to the method of our research team [5], we obtained the ethanol extract $86.0 \mathrm{~g}$ in the range of $30-60 \%$ as refined $n$-butanol extract with the root of dried PJ (1000 g). The HPLC-UV chromatogram of the refined $n$-butanol extract from the root of $\mathrm{PJ}$ is presented in Figure 1. Six major chromatogram peaks were marked. Four peaks among them were detected by comparing their retention time with those of the authentic standards. Peaks numbered 1-4 represent chikusetsusaponin V, Pjs-2, chikusetsusaponin IV, and chikusetsusaponin IVa, respectively. These four saponins are all oleanane type saponins. In our present extraction and purification method, the content of these four saponins detected by HPLC-UV analysis was $92.5 \%$. The other two peaks were unidentified (Figure 1).

\section{Changes of liver/body weight, food, and water intake}

No mice died during the 11 weeks of the experimental period. Figure 2 summarizes the effect of SPJ on body weight changes induced by the HF diet. After 11 weeks of exposure, the mean weight of the control group increased slowly, while in the model group it showed positive growth $(p<0.05)$. In contrast, with the SPJ treatment, the body weight increased slowly, especially in the SPJ high dose group $(p<0.05)$, compared to that in the model group (Figure 2).

Table II shows the food, water intake, final body weight and liver weight of different groups of mice. As time passed, the mice in the model group ate more food and drank more water compared

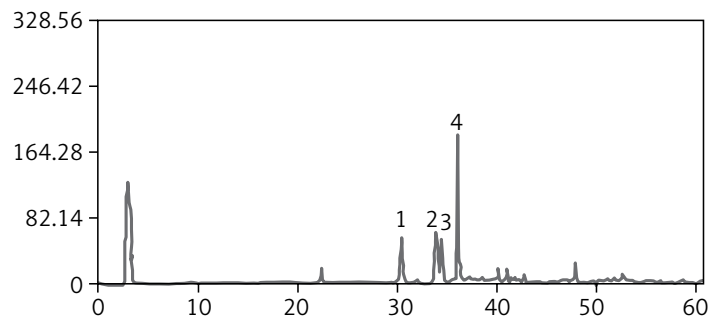

Figure 1. The saponins of Panax japonicus analyzed by HPLC-ELSD

1-chikusetsusaponin V, 2-Pjs-2, 3-chikusetsusaponin IV, 4 - chikusetsusaponin IVa. with the control group $(p<0.05)$. Administration of 100 and $300 \mathrm{mg} / \mathrm{kg}$ SPJ significantly reduced these values as compared with the model group $(p<0.05)$. By comparing the final body weight, liver weight and liver index, it was apparent that with the SPJ treatment the body weight and liver weight were markedly decreased.

\section{Changes of biochemical parameters in the sera}

In order to investigate the extent of liver injury following HF diet treatment, the serum levels of ALT, AST, TG, HDL, LDL and TBA were detected in the mice. The results showed that the activities of ALT and AST and the content of TG, LDL and TBA were obviously increased in the model group compared with those of the normal group, while the HDL content was decreased significantly ( $p<$ 0.01 . Also, in the SPJ low and high group the serum ALT and AST activities and the content of LDL, TG and TBA were significantly reduced $(p<0.01)$ compared with those of the model group, which suggested that treatment with SPJ had a beneficial effect on protecting mouse liver and reducing the lipid accumulation (Table III).

\section{Effect of SPJ on liver morphology and structure}

The liver appearance in the normal control group was normal color and smooth surface. While the liver appearance was very brittle and

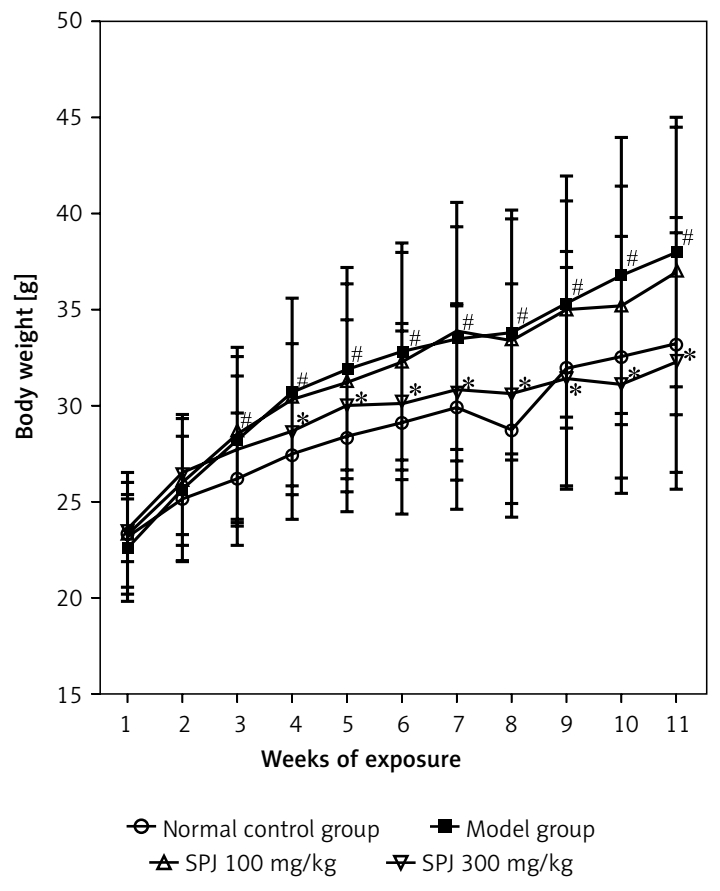

Figure 2. Changes of mice body weight. The data of body weight are presented as means \pm SD $(n=6)$

${ }^{*} p<0.01$ vs. the normal control group; ${ }^{*} p<0.01$ vs. the model group. 
Table II. Liver/body weight, food, and water intake of control and experimental animals

\begin{tabular}{|lcccc|}
\hline Parameter & Control & Model & \multicolumn{2}{c|}{ SPJ [mg/kg] } \\
\cline { 4 - 5 } & & & 100 & 300 \\
\hline Food intake [g/mouse/day] & $24.34 \pm 3.32$ & $31.90 \pm 4.78^{\#}$ & $26.50 \pm 5.23^{*}$ & $22.29 \pm 4.92^{*}$ \\
\hline Water intake [ml/mouse/day] & $31.28 \pm 2.02$ & $44.25 \pm 4.53^{\#}$ & $34.12 \pm 3.12^{*}$ & $33.13 \pm 3.90^{*}$ \\
\hline Final body weight [g] & $33.23 \pm 6.64$ & $38.09 \pm 7.03^{\#}$ & $37.10 \pm 7.49$ & $32.39 \pm 6.68^{*}$ \\
\hline Body weight net gain [g] & $9.91 \pm 4.17$ & $15.44 \pm 5.58^{\#}$ & $13.56 \pm 5.79^{*}$ & $8.82 \pm 5.81^{*}$ \\
\hline Body weight gain rate (\%) & $42.49 \pm 3.64$ & $68.17 \pm 5.38^{\#}$ & $57.60 \pm 4.38^{*}$ & $37.43 \pm 4.76^{*}$ \\
\hline Liver weight [g] & $4.24 \pm 0.93$ & $6.82 \pm 1.02^{\#}$ & $5.85 \pm 0.78$ & $3.74 \pm 0.67^{*}$ \\
\hline Liver index (\%) & $12.77 \pm 1.22$ & $17.90 \pm 1.25^{\#}$ & $15.78 \pm 1.73$ & $11.55 \pm 1.02^{*}$ \\
\hline
\end{tabular}

Table II shows the food, water intake, final body weight and liver weight of different groups of mice. They were increased compared with those in the control group $(p<0.05)$. Administration of 100 and $300 \mathrm{mg} / \mathrm{kg}$ SPJ significantly reduced these values as compared with the model group $(p<0.05)$. ${ }^{*} p<0.05$, compared with control group; ${ }^{*} p<0.05$, compared with model group.

Table III. Biochemical parameters in mice treated with SPJ

\begin{tabular}{|c|c|c|c|c|}
\hline \multirow[t]{2}{*}{ Parameter } & \multirow[t]{2}{*}{ Control } & \multirow[t]{2}{*}{ Model } & \multicolumn{2}{|c|}{ SPJ $[\mathrm{mg} / \mathrm{kg}]$} \\
\hline & & & 100 & 300 \\
\hline ALT & $39.00 \pm 7.85$ & $52.90 \pm 7.36^{\#}$ & $47.00 \pm 5.86$ & $42.50 \pm 6.90^{*}$ \\
\hline AST & $120.00 \pm 15.09$ & $150.78 \pm 18.60^{\#}$ & $111.71 \pm 12.51^{*}$ & $131.25 \pm 12.38^{*}$ \\
\hline HLD-C & $2.57 \pm 0.62$ & $1.76 \pm 0.49^{\#}$ & $2.690 \pm 0.71^{*}$ & $2.49 \pm 0.55^{\star}$ \\
\hline LDL-C & $0.10 \pm 0.02$ & $0.27 \pm 0.05^{\#}$ & $0.18 \pm 0.05^{\star}$ & $0.18 \pm 0.06^{*}$ \\
\hline TBA & $2.23 \pm 0.91$ & $4.44 \pm 1.14^{\#}$ & $2.40 \pm 1.10^{*}$ & $2.36 \pm 1.07^{*}$ \\
\hline TG & $0.63 \pm 0.06$ & $0.73 \pm 0.08^{\#}$ & $0.62 \pm 0.05^{*}$ & $0.47 \pm 0.07^{*}$ \\
\hline
\end{tabular}

\#p<0.05, compared with control group; $p<0.05$, compared with model group.

covered with particles on the surface in the model group, the liver in the SPJ low and high dose groups was significantly improved in appearance, being smoother (Figure $3 \mathrm{~A}$ ).

To detect the hepatoprotective effects of SPJ, we conducted histological examination of the extent of liver damage. Hepatic histopathology was assessed by staining paraffin sections of liver with $\mathrm{H}+\mathrm{E}$. The liver tissue showed normal architecture in the normal control group. In the model group, the structure of the hepatic cells was disordered, the liver lobule was fuzzy and even disappeared, hepatic cells showed obvious fatty degeneration and necrosis, and the liver portal area indicated hyperplastic fiber with infiltration of inflammatory cells. In the SPJ low and high dose groups the lipid degeneration and inflammatory response were significantly alleviated compared with the model group. Hepatocyte volume became smaller, hepatic lobules were clearly delineated, and the fat droplets were reduced (Figure $3 \mathrm{~B}$ ).

Under the electron microscope, the shape of the cellular mitochondria and endoplasmic reticulum was quite regular, the distributions of the mitochondria and endoplasmic reticulum were dense, and the arrangement of the mitochondrial crest was very tight in the normal control group. However, the liver tissue of the model group displayed a lot of fat vacuoles in the cells and was accompanied by a large area of damage and fracture of the mitochondria and the mitochondrial crest, while the endoplasmic reticulum amount decreased significantly. Whereas the hepatocyte lipid droplets in the SPJ low and high dose groups were markedly reduced, and the amount of the endoplasmic reticulum was obviously increased, the mitochondrial damage was apparently reduced. In addition, the mitochondrial crest was more developed and arranged distinctly orderly (Figure $3 \mathrm{C}$ ).

\section{Changes of collagen synthesis and deposition in the liver}

In the liver, collagens are the main components of the extracellular matrix (ECM), primarily composed of collagen I and III, which account for $95 \%$ of the total collagen in fibrotic liver. When the liver becomes fibrous, the basement membrane will be damaged, and the content of collagen can be increased significantly. We used Masson's trichrome staining, RT-PCR and western blotting to observe the influence of collagen fiber generation. 

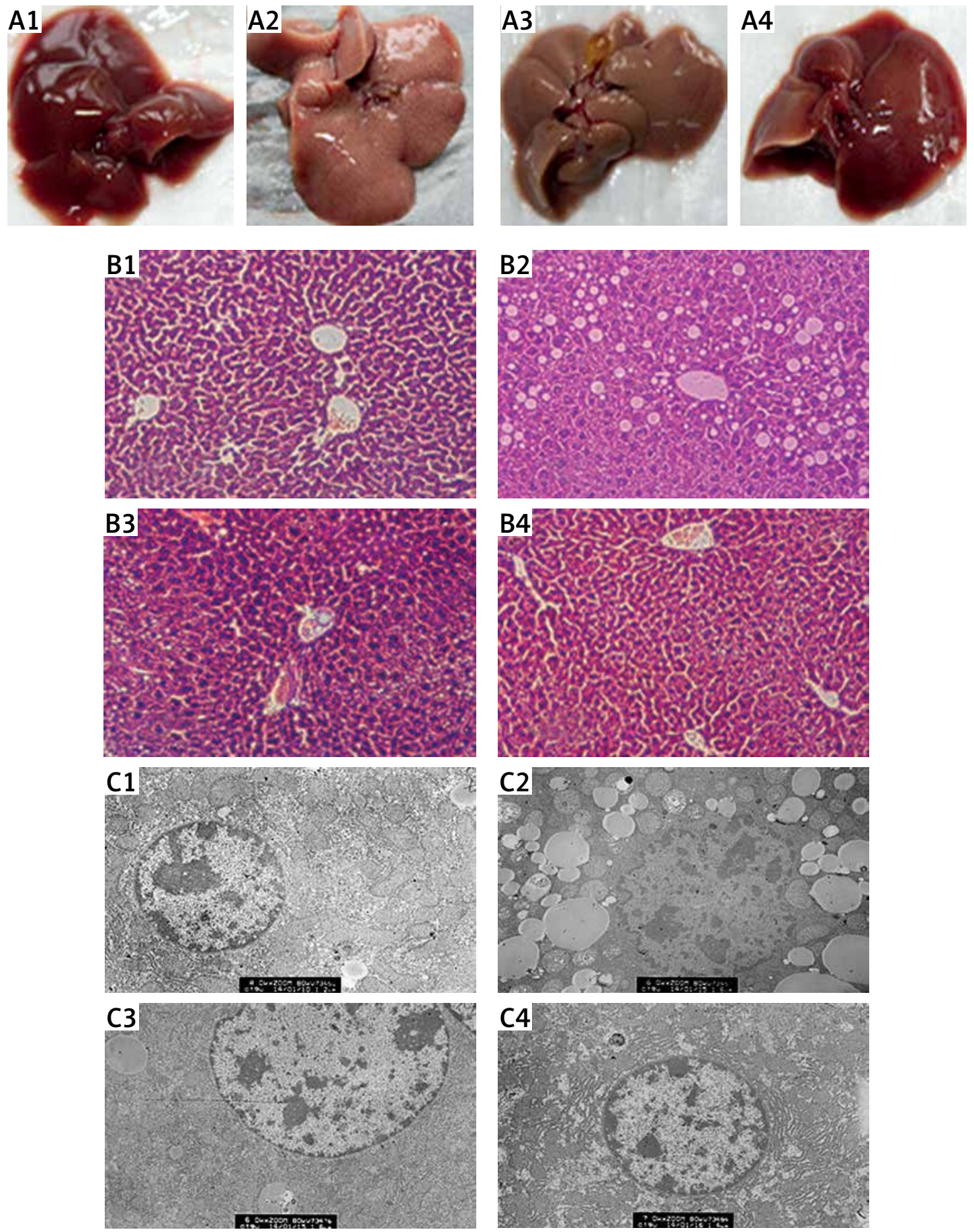

Figure 3. Pathological features of liver tissue. A - Morphologic changes in liver: (1) normal control group, liver appeared fresh, slippery and dense both in color and texture; (2) model group, on liver surface there appeared numerous tiny milky white particles; liver had rough appearance and grey color; (3) SPJ low dose group (100 mg/ $\mathrm{kg}$ ) and (4) SPJ high dose group (300 mg/kg), the liver appearance was improved dramatically, especially in the SPJ high dose group; the liver was redder, smoother and denser. B - H + E staining of liver tissue: (1) normal control group, showing a normal morphological structure in lobular architecture and hepatocytes; (2) model group, revealing conspicuous morphological disruption; (3) SPJ low dose group (100 mg/kg), displaying moderate improvement of morphological changes; (4) SPJ high dose group (300 mg/kg), demonstrating obvious improvement in the hepatocytes. Liver sections showed a dose-dependent reduction in the degree of liver injury. C - Ultrastructural observation of the hepatocytes: (1) normal control group, the cell ultrastructure was quite regular including mitochondria and endoplasmic reticulum; (2) model group, showing numerous fat vacuoles in the hepatocytes and accompanied by a large area of damage and fracture of the mitochondria and the mitochondrial crest; (3) SPJ low dose group $(100 \mathrm{mg} / \mathrm{kg})$, revealing some mitochondria and fewer fat vacuoles in the hepatocytes; (4) SPJ high dose group (300 mg/kg), revealing almost normal mitochondria and endoplasmic reticulum in the cellular ultrastructure 1 - normal control group, 2-model group, 3-SPJ $100 \mathrm{mg} / \mathrm{kg}, 4$ - SPJ $300 \mathrm{mg} / \mathrm{kg}$. 
The results of Masson's staining showed that the perivascular collagen fiber was dyeing only in the portal area in the normal group (Figure $4 \mathrm{~A}$ ). Compared with the normal control group, the hepatic portal area and portal vein in the model group manifested obviously increasing collagen fibers, while around the central vein the hyper-
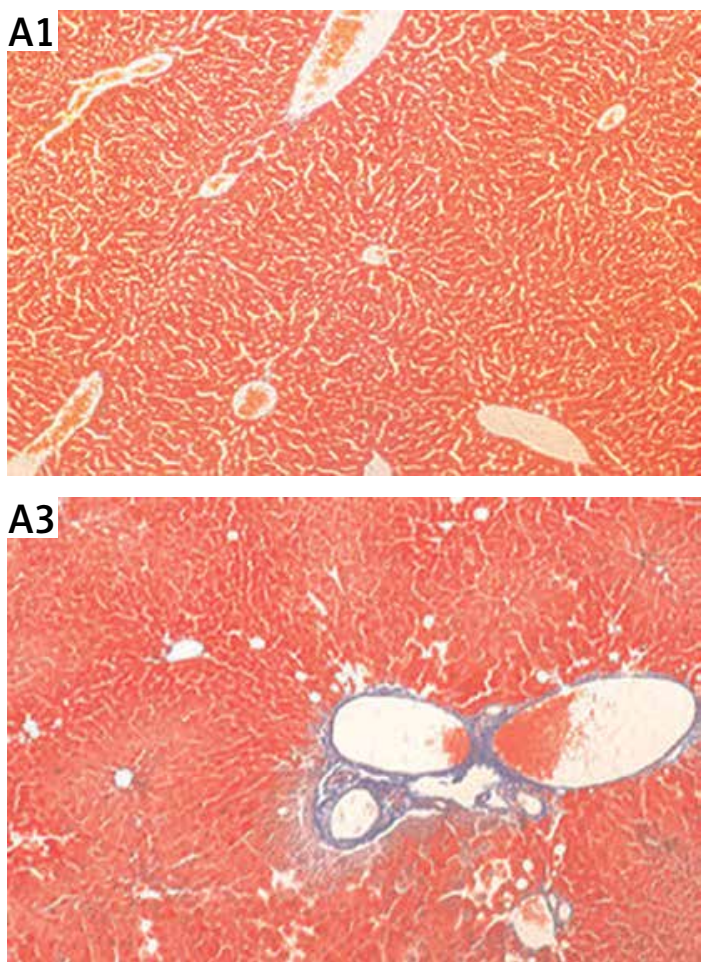

B

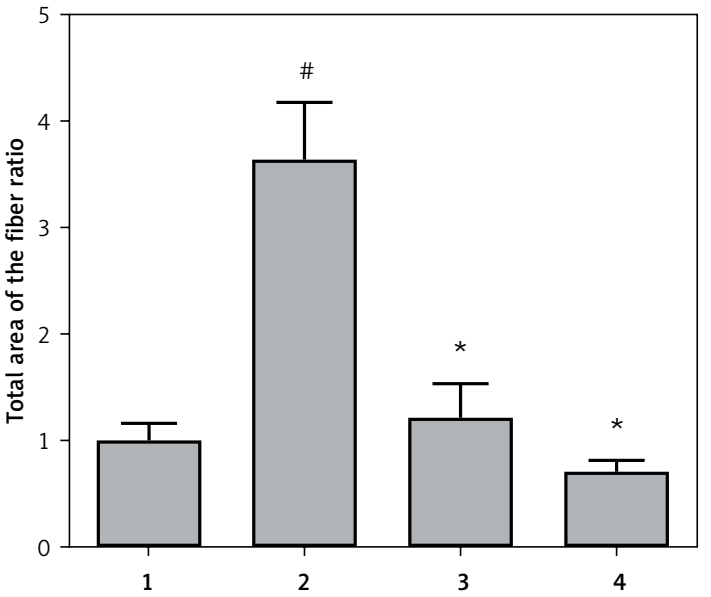

plastic collagen fibers were distributed along the hepatic sinus and connected to each other, then they formed the diaphragm as a connection to the portal area and the central vein. Treatment with SPJ in the low and high dose groups significantly inhibited the production of collagen fibers, while collagen fiber hyperplasia only appeared in the
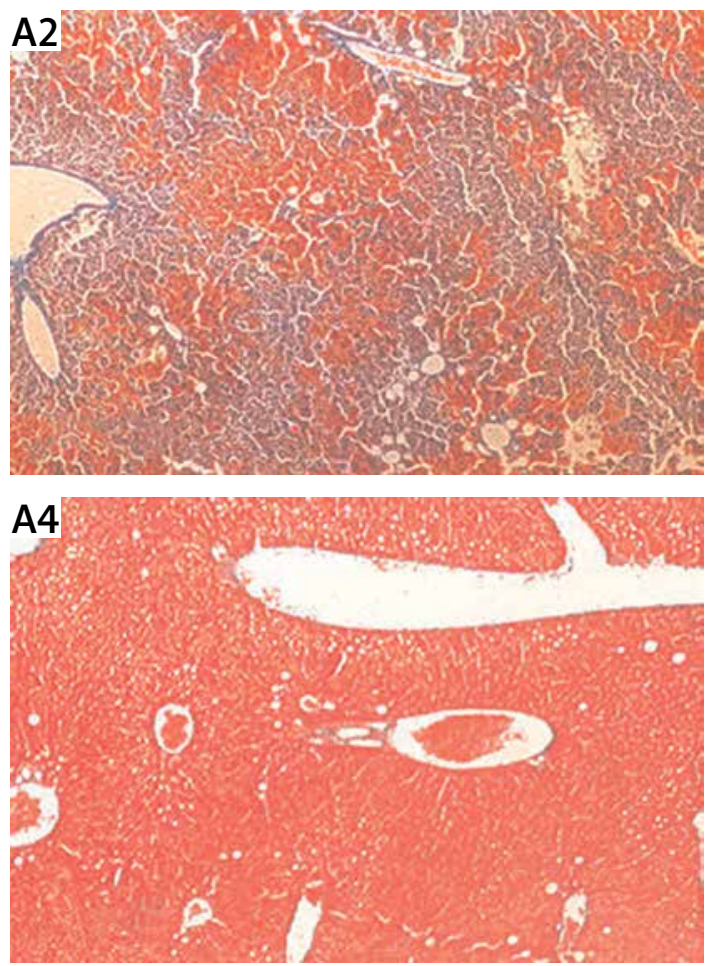

C

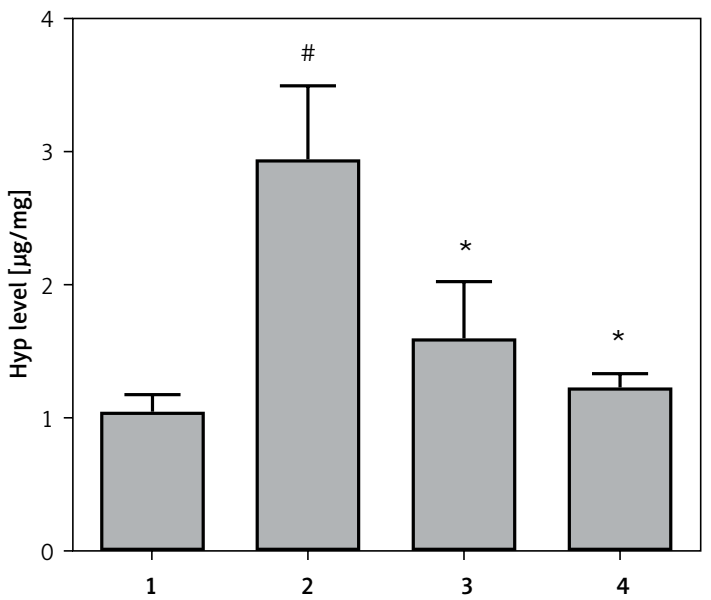

Figure 4. SPJ treatment inhibited collagen deposition in the mouse liver. A - Masson's trichrome staining of liver tissues (magnification, 200x): (1) normal control group, normal structure of the hepatic lobule; (2) model group, extensive accumulation of continuous fibrotic septa was around the central vein in the liver; (3) SPJ low dose group (100 mg/kg), showing less collagen deposited and pseudolobule formation; (4) SPJ high dose group (300 mg/kg), collagen fiber rarely existed. B - Relative quantitative analysis of the fibrosis in Masson staining: using software analyses the fiber sensitivity, in the fatty liver fibrosis group, the sum area of the fiber was raised evidently; SPJ $(100 \mathrm{mg} / \mathrm{kg}$ and $300 \mathrm{mg} / \mathrm{kg}$ ) groups showed a distinctly smaller sum area of the fiber; the sum area of the fiber ratio is presented as means $\pm S D(n=6)$. ${ }^{*} p<0.01$ vs. normal control group; ${ }^{*} p<0.01$ vs. model group. $C-$ Hyp content detection in the liver tissue: compared with the model group, the Hyp content sharply declined with the SPJ treatment $(100 \mathrm{mg} / \mathrm{kg}$ and $300 \mathrm{mg} / \mathrm{kg})$; the Hyp content is presented as means $\pm \mathrm{SD}(n=6) . \# p<0.01 \mathrm{vs}$. normal control group; ${ }^{*} p<0.01$ vs. model group 
D
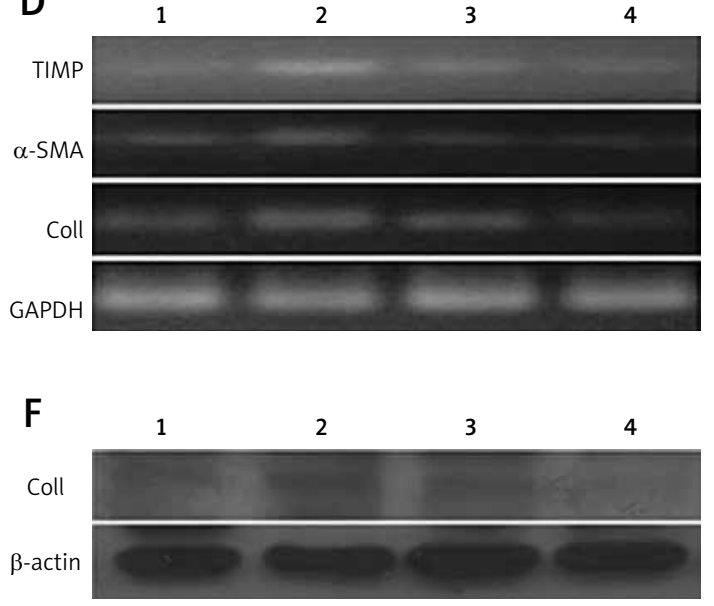

G

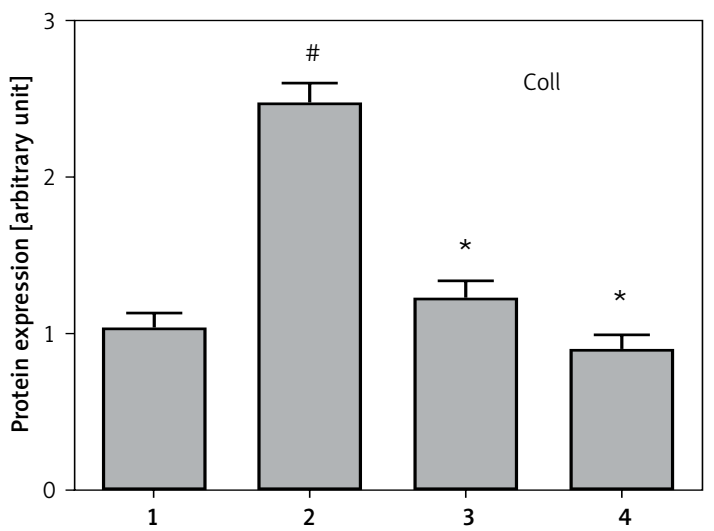

portal area. Through the analysis of the results of Masson staining by the Imager-Pro Plus 7.0C software, the sum area of the fiber in the model group was 2.43 fold higher compared with the normal control group $(p<0.01)$, while in the SPJ $(100 \mathrm{mg} /$ $\mathrm{kg}, 300 \mathrm{mg} / \mathrm{kg}$ ) groups it significantly decreased by about $74 \%$ and $86 \%$ by contrast with the model group, respectively $(p<0.01)$ (Figure $4 \mathrm{~B})$.

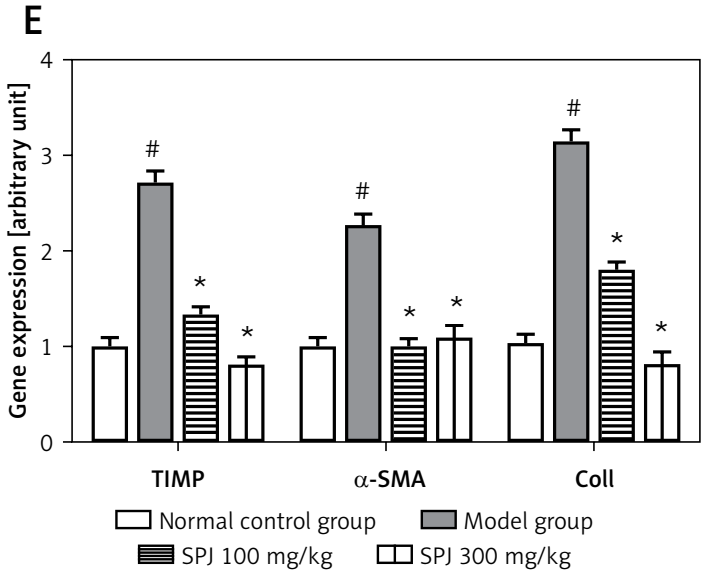

Figur 4. D and $E$ - Effect of SPJ on the expression of TIMP, $\alpha$-SMA, COLL l $\alpha$ in the liver tissues: SPJ treatment $(100 \mathrm{mg} / \mathrm{kg}$ and $300 \mathrm{mg} / \mathrm{kg})$ reduced TIMP, $\alpha$-SMA, COLL l $\alpha$ gene expression compared with liver fibrosis group; however, in the SPJ high dose group $(300 \mathrm{mg} / \mathrm{kg}$ ) the decrease was more obvious; the gene expression levels are presented as means $\pm \mathrm{SD}(n=6) .{ }^{*} p<0.01$ vs. normal control group; ${ }^{*} p<$ 0.01 vs. model group. $\mathbf{F}$ and $\mathbf{G}-$ Expression of COLL $1 \alpha$ protein in the liver tissues: with SPJ treatment especially the high dose group $(300 \mathrm{mg} / \mathrm{kg}$ ) significantly downregulated levels of COLL l $\alpha$ compared with the model group; the protein expression levels are presented as means $\pm \mathrm{SD}(n=6) .{ }^{*} p<0.01$ vs. normal control group; ${ }^{*} p<0.01$ vs. model group

1 - normal control group, 2 - model group, 3 - SPJ $100 \mathrm{mg} / \mathrm{kg}, 4$ - SPJ $300 \mathrm{mg} / \mathrm{kg}$.

The METAVIR [6] and Brunt [7] methods were used to classify the severity of liver fibrosis. As shown in Table IV, for the normal group, the scores were mainly concentrated within $\mathrm{FO}-\mathrm{F} 3$, the most in FO, while the scores of the model group were principally focused in F1-F3 and there were zero scores of FO in both METAVIR and BRUNT. With the SPJ treatment in low and high dose groups, the

Table IV. Effects of SPJ on fatty liver fibrosis score

\begin{tabular}{|lccccccc|}
\hline Group & Method & F0 & F1 & F2 & F3 & F4 & Liver fibrosis area (\%) \\
\hline Control & METAVIR & 8 & 4 & 0 & 0 & 0 & $24.86 \pm 8.30$ \\
\cline { 2 - 7 } & Brunt & 8 & 3 & 1 & 0 & 0 & $33.97 \pm 1.74$ \\
\hline Model & METAVIR & 0 & 6 & 4 & 2 & 0 & $86.46 \pm 6.50^{*}$ \\
\cline { 2 - 7 } & Brunt & 0 & 2 & 4 & 6 & 0 & $135.24 \pm 42.54^{\#}$ \\
\hline SPJ $100 \mathrm{mg} / \mathrm{kg}$ & METAVIR & 3 & 6 & 2 & 1 & 0 & $60.78 \pm 14.48^{*}$ \\
\hline SPJ $300 \mathrm{mg} / \mathrm{kg}$ & Brunt & 3 & 3 & 3 & 3 & 0 & $86.16 \pm 15.78^{*}$ \\
& METAVIR & 4 & 6 & 1 & 1 & 0 & $53.47 \pm 19.65^{*}$ \\
\hline & Brunt & 4 & 4 & 2 & 2 & 0 & $67.94 \pm 7.53^{*}$ \\
\hline
\end{tabular}

Brunt and METAVIR scoring systems were applied on a scale of 0-4: FO, no fibrosis; F1, portal fibrosis without septa (bridge of connective tissue between two portal tracts, a portal tract and a centrolobular vein, or between two centrolobular veins); F2, few septa; F3, numerous septa without cirrhosis; F4, cirrhosis (liver tissue is mutilated by nodular fibrosis that delineates hepatocyte nodules). For statistical analysis, the FO and F1 were considered insignificant (minimal or no) fibrosis, while the scores F2-F3 were considered significant fibrosis and cirrhosis as stage F4. ${ }^{*} p<0.05$, compared with control group; ${ }^{*} p<0.05$, compared with model group. 
scores were gradually close to the normal group. Also, the liver fibrosis area decreased significantly with SPJ compared with the model group. Thus, it was extremely clear that SPJ could improve the situation of liver fibrosis.

Hydroxyproline, as an index of connective tissue turnover, could reflect the metabolism of the collagen. Hyp content in the liver specimens represented the total amount of collagen in livers, which was quantified to evaluate the degree of liver fibrosis by using a colorimetric method. The results indicated that the Hyp content was $189 \%$ higher in the model group compared with the normal control group $(p<0.01)$. By contrast, the SPJ $(100 \mathrm{mg} /$ $\mathrm{kg}, 300 \mathrm{mg} / \mathrm{kg}$ ) groups showed a decrease of $46 \%$ and $59 \%$, respectively $(p<0.01)$ (Figure 4 C).

The mRNA fragments specific to TIMP, $\alpha$-SMA and Coll were amplified for the fibrosis markers by RT-PCR. The densitometric analysis of the fragments was normalized against the corresponding GAPDH transcript. Gene expression of TIMP, $\alpha$-SMA and Coll in the model group was $174 \%$, $128 \%$ and $217 \%$ higher, respectively, than that in the normal control group; treatment with SPJ (100 mg/kg) caused a decrease of $51 \%, 57 \%$ and $44 \%$, respectively, in mRNA expression compared with the model group; also treatment with SPJ $(300 \mathrm{mg} / \mathrm{kg})$ caused a $71 \%, 52 \%$ and $74 \%$ decrease respectively. The decrease in mRNA expressions of TIMP, $\alpha$-SMA and Coll was significant compared with the model group $(p<0.01)$ (Figures $4 \mathrm{D}$ and $\mathrm{E}$ ). Protein expression of Coll in the fatty liver fibrosis group was $211 \%$ higher than that in the normal control group; treatment with SPJ (100 mg/kg) and SPJ (300 mg/kg) caused a decrease of $51 \%$ and $64 \%$, respectively, in protein expression compared with the fibrosis group. The decrease in protein expression of Coll was significant compared with the model group $(p<0.01)$ (Figures $4 \mathrm{~F}$ and $\mathrm{G}$ ). All of these results suggest that the liver fibrosis model is successful and the SPJ can protect against liver injury.

\section{Effects of SPJ on the genes and proteins associated with endoplasmic reticulum stress (ERS)}

GRP78 and CHOP are the classic markers of ERS. For a comprehensive evaluation of the effects of SPJ against fatty liver fibrosis, the expression of some related proteins or genes was investigated. The results showed that the gene expression of GRP78 and CHOP in the fatty liver fibrosis group was $756 \%$ and $40 \%$ higher, respectively, than that in the normal group; treatment with SPJ $(100 \mathrm{mg} /$ $\mathrm{kg}$ ) caused a decrease of $67 \%$ and $78 \%$, respectively, in mRNA expression compared with the model group; treatment with SPJ $(300 \mathrm{mg} / \mathrm{kg}$ ) caused a $71 \%$ and $69 \%$ decrease respectively. The decrease in mRNA expression of GRP78 and CHOP was significant compared with the model group $(p<0.01)$ (Figure $5 \mathrm{~A}$ ).

Meanwhile the protein expression of GRP78 in the model group caused an increase of $153 \%$ compared with the normal group. In contrast, in the SPJ low and high dose groups, the protein expression of GRP78 caused a decrease of $69 \%$, which was significant compared with the model group ( $p<0.01$ ) (Figure 5 B). The phosphorylation of JNK was compared with total JNK, which was $59 \%$ and $53 \%$ lower in the SPJ $(100 \mathrm{mg}$ / $\mathrm{kg}, 300 \mathrm{mg} / \mathrm{kg}$ ) compared with the model group $(p<0.01)$ (Figure $5 \mathrm{C}$ ).

\section{Discussion}

Animal models have been used for several decades to study liver diseases and evaluate drug activity $[8,9]$. An enormous number of methods can be used to establish the liver fibrosis model in mouse; carbon tetrachloride $\left(\mathrm{CCl}_{4}\right)[10,11]$, thioacetamide (TAA) [12, 13], and dimethyl or diethyl nitrosamine (DMN or DEN) [14-16] are the most commonly used hepatotoxic agents in the classical models of bridging fibrosis. In addition, biliary fibrosis, immunologically induced fibrosis, alcohol-induced fibrosis and NASH-associated fibrosis were quite common ways of building liver fibrosis models. Recently, a combined model of HF diet and gold thioglucose (GTG) administration was reported [17]. Gold thioglucose is known to induce lesions in the ventromedial hypothalamus, leading to hyperphagia and obesity. Gold thioglucose was administered intraperitoneally to BABL/c mice, and thereafter they were fed an HF diet for 12 weeks. As a result, obesity with increased abdominal adiposity, glucose intolerance, insulin resistance, and steatohepatitis with hepatocyte ballooning, $\mathrm{MH}$, and pericellular fibrosis were induced. So we chose a combined method to establish the fatty liver fibrosis model, which includes an HF diet and a porcine serum immunological agent which imitates hepatic inflammation. Researchers reported that this combined method could shorten the modeling time and reduce the death of the animal, and was closer to the clinical pathological damage of hepatic fibrosis etiology and development [18].

Activities of serum ALT and AST were conventionally used as biochemical markers to assess liver injury [19]. In the present study, a long-term $\mathrm{HF}$ diet combined with intraperitoneal injections of xenogeneic protein resulted in a significant elevation of serum ALT and AST. Administration of SPJ markedly attenuated the abnormal activities of serum ALT and AST, which indicated that SPJ has potent hepatoprotective effects. Meanwhile a significant reduction in TG, HDL, and TBA and an elevation in LDL were observed following treat- 

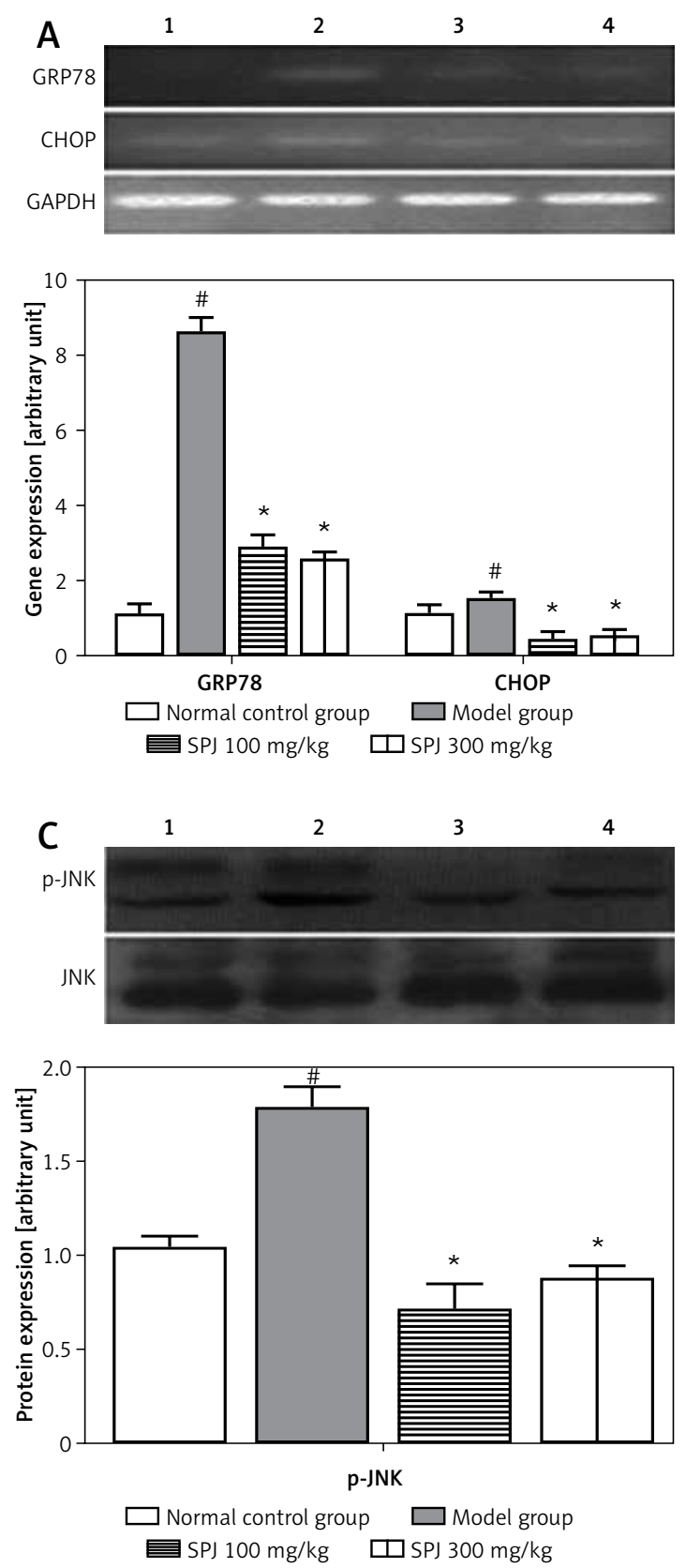

ment with SPJ. Therefore, we hypothesized that SPJ exerts a protective effect on fatty liver fibrosis.

It is known that the destruction of liver structure and deposition of collagen fiber are vital biomarkers in the development of liver fibrosis [20]. Previous studies revealed that TIMP inhibits collagenase activity. Recent studies [20-22] revealed an anti-fibrogenesis effect caused by SPJ reducing the expression of $\alpha$-SMA and TIMP. Collagens are the main components of the ECM. Collagen I is one of the main components of collagen, while collagen is a major component of connective tissue. Both the protein and the gene expression levels of Coll were reduced evidently by SPJ. These
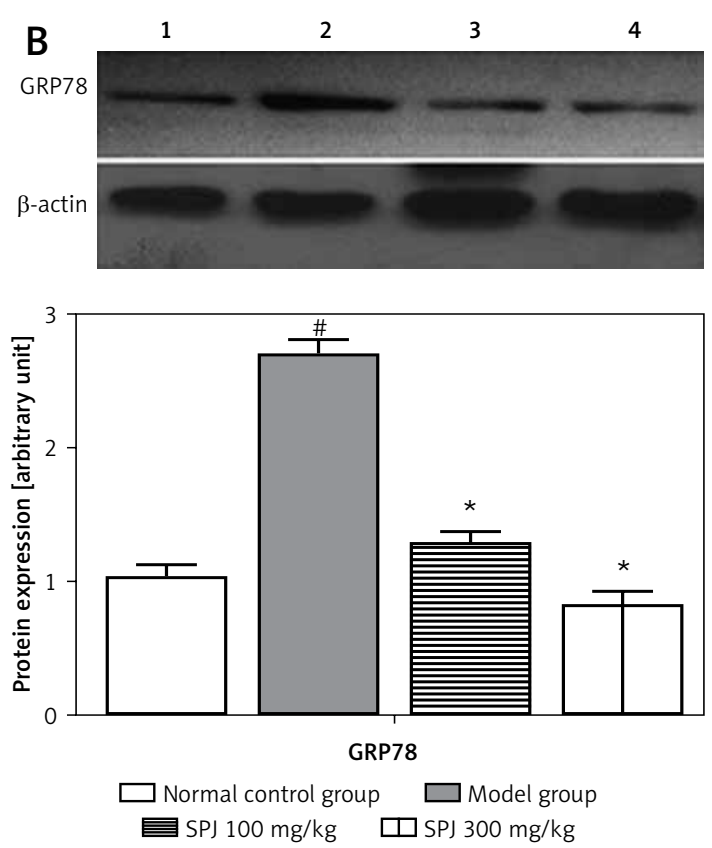

Figure 5. SPJ treatment recovered the expression of ERS-associated genes and proteins. A - Effects of SPJ on the gene expression of GRP78 and CHOP in the liver tissues: in SPJ $(100 \mathrm{mg} / \mathrm{kg}$ and $300 \mathrm{mg} / \mathrm{kg})$, the mRNA levels of GRP78 and CHOP were remarkably decreased compared with the model group, the gene expression levels are presented as means $\pm \mathrm{SD}(n=6) .{ }^{*} p<0.01$ vs. normal control group; ${ }^{*} p<0.01$ vs. model group. $\mathbf{B}-$ Effects of SPJ on protein expression of GRP78 in the liver tissues: anti- $\beta$-actin blotting was used as control for equal protein loading. Compared with the model group, SPJ $(100 \mathrm{mg} / \mathrm{kg}$ and $300 \mathrm{mg} / \mathrm{kg})$ treatment significantly suppressed the protein expression of GRP78; the protein expression levels are presented as means \pm SD $(n=6) .{ }^{\#} p<0.01$ vs. normal control group; ${ }^{*} p<0.01$ vs. model group. $\mathrm{C}$ - Effects of SPJ on the protein expression of JNK and $p$-JNK in the liver tissues: Noticeable changes of the JNK protein expression did not appear in the normal control group, model group and SPJ (100 mg/kg and $300 \mathrm{mg} /$ $\mathrm{kg}$ ) groups, while the expression of p-JNK was significantly decreased in SPJ $(100 \mathrm{mg} / \mathrm{kg}$ and $300 \mathrm{mg} / \mathrm{kg})$ groups in contrast with the model group; protein expression levels are presented as means \pm SD $(n=6) .{ }^{\#} p<0.01$ vs. normal control group; ${ }^{*} p<0.01$ vs. model group

1 - normal control group, 2 - model group, 3 - SPJ $100 \mathrm{mg} / \mathrm{kg}$, 4 - SPJ $300 \mathrm{mg} / \mathrm{kg}$.

results indicated that SPJ may reduce the generation of collagen fiber. Through the detection of Hyp content in the liver tissues, we found that SPJ resulted in a reduction of Hyp content. In addition, we observed through Masson's staining that the production of collagen fibers was significantly inhibited by the treatment with SPJ.

ERS has emerged as a common feature relevant to the pathogenesis of diseases associated with liver fibrosis [23]. The ERS-related factor GRP78, as an endoplasmic reticulum steady-state receptor, is one of the key factors to start and regulate the endoplasmic reticulum steady state. To some extent, the expression of GRP78 can reflect the adjustment ability to 
start and regulate the endoplasmic reticulum steady state [24]. CHOP is apoptosis signaling molecule induced by ERS, plays an important role in cell death, and is the direct result of the ERS apoptosis response. In recent years, research has shown that the JNK signaling pathway could mediate cell apoptosis through ERS $[25,26]$. In the present study, it was found that GRP78, CHOP and p-JNK were up-regulated in the model and down-regulated by SPJ.

In conclusion, our results showed that the total saponins of Panax japonicus had a potential protective effect against the HF diet combined with intraperitoneal injection of pig serum induced fatty liver fibrosis via inhibition of the endoplasmic reticulum stress response and CHOP and JNK-mediated apoptosis pathways and inflammation pathways. However, further studies should be carried out to investigate in depth the mechanisms and drug targets of fatty liver fibrosis.

\section{Acknowledgments}

This work was supported by grants from the National Nature and Science Foundation of China (NSFC) (No. 81273895) and the Foundation for Innovative Research Groups of the Hubei Province Natural Science Foundation of China (No. 2013CFA014) to Dr. Yuan Ding.

\section{Conflict of interest}

The authors declare no conflict of interest.

\section{References}

1. Dyson J, Day C. Treatment of non-alcoholic fatty liver disease. Dig Dis 2014; 32: 597-604.

2. Paschos P, Paletas K. Non alcoholic fatty liver disease and metabolic syndrome. Hippokratia 2009; 13: 9-19.

3. Lu Y, Wei J, Tang Y, et al. Evaluation of fatty liver fibrosis in rabbits using real-time shear wave elastography. Exp Ther Med 2014; 8: 355-62.

4. Balunas MJ, Kinghorn AD. Drug discovery from medicinal plants. Life Sci 2005; 78: 431-41.

5. He YM, Lu KM, Yuan D, Zhang CC. Studies on preparative technology and quantitative determination for extracts of total saponin in roof of Panax japonicus. Zhongguo Zhong Yao Za Zhi 2008; 33: 2607-11.

6. Elesawy BH, Abd El Hafez A, Dorgham LS, El-Askary A. Limited reliability of five non-invasive biomarkers in predicting hepatic fibrosis in chronic HCV mono-infected patients opposed to METAVIR scoring. Pathol Res Pract 2014; 210: 922-8.

7. Merat S, Khadem-Sameni F, Nouraie M, et al. A modification of the Brunt system for scoring liver history of patients with non-alcoholic fatty liver disease. Arch Iran Med 2010; 13: 38-44.

8. Tsukamoto H, Matsuoka M, French SW. Experimental models of hepatic fibrosis: a review. Semin Liver Dis 1990; 10: 56-65.

9. Iredale JP. Models of liver fibrosis: exploring the dynamic nature of inflammation and repair in a solid organ. J Clin Invest 2007; 117: 539-48.
10. Kumar P, Yang HJ, Lee JY, et al. Adiponectin agonist ADP355 attenuates $\mathrm{CCl} 4$-induced liver fibrosis in mice. PLoS One 2014; 9: e110405.

11. Guo G, Wu H, Liu M, Ding C, Qin J, Yang X. Differential proteome analysis of carbon tetrachloride-induced mouse liver fibrosis. Sheng Wu Gong Cheng Xue Bao 2014; 30: 1105-14.

12. Al-Attar AM. Attenuating effect of Ginkgo biloba leaves extract on liver fibrosis induced by thioacetamide in mice. J Biomed Biotechnol 2012; 2012: 761450.

13. Mohamed SN, Zhuo L. Imidazolium salt attenuates thioacetamide-induced liver fibrosis in mice by modulating inflammation and oxidative stress. Dig Liver Dis 2012; 44: 665-73.

14. Hu QW, Liu GT. Effects of bicyclol on dimethylnitrosamine-induced liver fibrosis in mice and its mechanism of action. Life Sci 2006; 79: 606-12.

15. Zheng JF, Liang LJ, Wu CX, Chen JS, Zhang ZS. Transplantation of fetal liver epithelial progenitor cells ameliorates experimental liver fibrosis in mice. World J Gastroenterol 2006; 12: 7292-8.

16. Kim KS, Yang HJ, Lee JY, et al. Effects of beta-sitosterol derived from Artemisia capillaris on the activated human hepatic stellate cells and dimethylnitrosamine-induced mouse liver fibrosis. BMC Complement Altern Med 2014; 14: 363.

17. Ogasawara $M$, Hirose $A$, Ono $M$, et al. A novel and comprehensive mouse model of human non-alcoholic steatohepatitis with the full range of dysmetabolic and histological abnormalities induced by gold thioglucose and a high-fat diet. Liver Int 2011; 31: 542-51.

18. Wu W, Liu YJ, Chen DT, Zou XH, Liu CQ. Experimental studies of high fat diet combined with xenogeneic serum induced liver fibrosis model in mice. Modern Immunol 2014; 33: 216-9.

19. Sturgill MG, Lambert GH. Xenobiotic-induced hepatotoxicity: mechanisms of liver injury and methods of monitoring hepatic function. Clin Chem 1997; 43: 1512-26.

20. Arthur MJ, Mann DA, Iredale JP. Tissue inhibitors of metalloproteinases, hepatic stellate cells and liver fibrosis. J Gastroenterol Hepatol 1998; 13: S33-8.

21. Lichtinghagen R, Michels D, Haberkorn Cl, et al. Matrix metalloproteinase (MMP)-2, MMP-7, and tissue inhibitor of metalloproteinase-1 are closely related to the fibroproliferative process in the liver during chronic hepatitis C. J Hepatol 2001; 34: 239-47.

22. Zhang $\mathrm{C}$, Wang $\mathrm{Y}$, Chen $\mathrm{H}$, et al. Protective effect of the herbal medicine Gan-fu-kang against carbon tetrachloride-induced liver fibrosis in rats. Mol Med Rep 2013; 8: 954-62.

23. Li X, Wang Y, Wang H, Huang C, Li J. Endoplasmic reticulum stress is the crossroads of autophagy, inflammation, and apoptosis signaling pathways and participates in liver fibrosis. Inflamm Res 2015; 64: 1-7.

24. Keegan A, Morecroft I, Smillie D, Hicks MN, Maclean MR. Contribution of the $5-\mathrm{HT}$ (1B) receptor to hypoxia-induced pulmonary hypertension: converging evidence using 5-HT (1B)-receptor knockout mice and the 5-HT (1B/1D)-receptor antagonist GR127935. Circ Res 2001; 89: 1231-9.

25. Manning AM, Davis RJ. Targeting JNK for therapeutic benefit: from junk to gold? Nat Rev Drug Discov 2003; 2: $554-65$

26. Xu C, Maitre BB, Reed JC. Endoplasmic reticulum stress: cell life and death decisions. J Clin Invest 2005; 115: 2656-64. 